\title{
Emerging serotype III sequence type 17 group B streptococcus invasive infection in infants: the clinical characteristics and impacts on outcomes
}

Yi Kao ${ }^{1,4+}$, Ming-Horng Tsai ${ }^{2,4 \dagger}$, Mei-Yin Lai ${ }^{1,4}$, Shih-Ming Chu ${ }^{1,4}$, Hsuan-Rong Huang ${ }^{1,4}$, Ming-Chou Chiang ${ }^{1,4}$, Ren-Huei $\mathrm{Fu}^{1,4}$, Jang-Jih $\mathrm{Lu}^{3,4,5}$ and Jen-Fu Hsu ${ }^{1,4,6^{*}}$

\begin{abstract}
Background: Group B Streptococcus (GBS) is an important pathogen that causes high mortality and morbidity in young infants. However, data on clinical manifestations between different GBS serotypes and correlation with molecular epidemiology are largely incomplete. The aim of this study was to determine the serotype distribution, antimicrobial resistance, clinical features and molecular characteristics of invasive GBS isolates recovered from Taiwanese infants.

Methods: From 2003 to 2017, 182 non-duplicate GBS isolates that caused invasive disease in infants less than one year of age underwent serotyping, multilocus sequence typing (MLST) and antibiotic susceptibility testing. The clinical features of these infants with GBS disease were also reviewed.

Results: Of the 182 patients with invasive GBS disease, 41 (22.5\%) were early-onset disease, 121 (66.5\%) were lateonset disease and 20 (11.0\%) were late late-onset disease (>90 days of age). All these patients were treated with effective antibiotics on time. Among them, 51 (28.0\%) had meningitis, 29 (16.0\%) had neurological complications, $12(6.6 \%)$ died during hospitalization, and 15 (8.8\%) out of 170 patients who survived had long-term neurological sequelae at discharge. Serotype III GBS strains accounted for 64.8\%, followed by serotype la (18.1\%) and Ib (8.2\%). MLST analysis revealed 11 different sequence types among the 182 isolates and ST-17 was the most dominant sequence type (56.6\%). The correlation between serotype III and ST17 was evident, as ST17 accounted for $87.3 \%$ of all serotype III isolates. There was an obvious increasing trend of type III/ST-17 GBS that caused invasive disease in infants. All isolates were susceptible to penicillin, cefotaxime, and vancomycin, while 68.1 and $65.9 \%$ were resistant to erythromycin and clindamycin, respectively.
\end{abstract}

Conclusions: Despite timely and appropriate antibiotic treatment, a significant proportion of invasive GBS disease still inevitably causes adverse outcomes. Further study to explore preventive strategies and development of serotype-based vaccines will be necessary in the future.

Keywords: Group B streptococcus, Bacteremia, Drug resistance, Invasive streptococcal infection, Epidemiology

\footnotetext{
* Correspondence: jeff0724@gmail.com

${ }^{\dagger}$ Yi Kao and Ming-Horng Tsai contributed equally to this work.

'Division of Pediatric Neonatology, Department of Pediatrics, Chang Gung

Memorial Hospital, Taoyuan, Taiwan

${ }^{4}$ College of Medicine, Chang Gung University, Taoyuan, Taiwan

Full list of author information is available at the end of the article
}

(c) The Author(s). 2019 Open Access This article is distributed under the terms of the Creative Commons Attribution 4.0 International License (http://creativecommons.org/licenses/by/4.0/), which permits unrestricted use, distribution, and reproduction in any medium, provided you give appropriate credit to the original author(s) and the source, provide a link to the Creative Commons license, and indicate if changes were made. The Creative Commons Public Domain Dedication waiver (http://creativecommons.org/publicdomain/zero/1.0/) applies to the data made available in this article, unless otherwise stated. 


\section{Background}

Group B Streptococcus (GBS) or Streptococcus agalactiae is a Gram-positive coccus found in 15 to $30 \%$ of healthy women as part of normal gastrointestinal and genital tract flora [1-4]. GBS can cause invasive diseases in newborns after vertical transmission, in the elderly persons and those who have underlying immunocompromised status [5-7]. Invasive GBS infections in infants are of great concern, because a significant proportion of infants may have life-threatening meningitis, pneumonia, and bacteremia [7-11]. Recent studies also found poor long-term outcomes in GBS-infected infants, including an increased risk of death and chronic morbidities such as cerebral palsy, epilepsy, and various neurological sequelae [10-12].

Invasive GBS strains have a major virulence factor in the polysaccharide capsule, and 10 recognized capsular polysaccharides serotypes (CPS) have been identified by current molecular method, i.e., serotype Ia, Ib, and II to IX $[8,9]$. Because the GBS serotype distributions and carriage rates vary greatly in different geographical areas, and the GBS carriage varies temporally $[13,14]$, it is important to have local and updated information regarding the molecular epidemiology of GBS strains to develop vaccines and optimize the implementation of GBS prevention algorithm [15-17]. In Taiwan, routine GBS screening has been carried out among pregnant women since 2012 and the intrapartum prophylaxis of GBS for pregnant women was also implemented in our institute since 2012. In order to obtain more precise clinical, epidemiological and molecular characteristics of invasive GBS disease, we retrospectively collected data from infants aged less than 1 year with culture-proven invasive GBS disease from the largest medical center in Taiwan. The results of serotyping, clinical characteristics, multilocus sequence typing (MLST) analysis of these GBS isolates and antimicrobial susceptibility were reported in this study.

\section{Methods}

\section{GBS isolates, data collection and definition}

Between January 2003 and December 2017, all young infants aged less than one year with invasive GBS diseases were enrolled and their data were retrieved retrospectively from the database of Chang Gung Memorial Hospital $(\mathrm{CGMH})$. This database was filled by the clinicians. We reviewed the electronic chart records for patients' demographics, clinical characteristics, treatment and outcomes. All GBS isolates were obtained from the bacterial library of CGMH's central laboratory. In our institute, all positive GBS cultures were collected before antibiotics were given to infants. Early-onset disease (EOD) and late-onset disease (LOD) were defined as disease occurred between 0 and 7 days of age and between
8 and 90 days of age $[6,11,12]$, respectively. Disease occurred after 90 days of age was classified as late LOD (LLOD) $[6,11,12]$. Invasive GBS disease was defined as GBS infection with GBS strains isolated from a sterile site, including blood, urine, cerebrospinal fluid (CSF), soft tissues (necrotic tissues, abscesses, or cellulitis), pleural or peritoneal fluids. We did not enroll GBS isolates from sputum or bronchoalveolar lavage fluid. This study was approved by the ethics committee of CGMH (IRB No. 104-6818B), and a waiver of informed consent for anonymous data collection was approved.

Meningitis was defined by the World Health Organization as the presence of clinical signs of possible serious bacterial infection [18] and CSF culture positive for bacterial pathogens or blood culture/polymerase chain reaction (PCR)/latex agglutination positive for bacterial pathogens with a CSF leukocyte count $>20 \times 10^{6} /$ L. Episodes reported by physicians with negative CSF cultures were also included if CSF results showed at least one individual marker of bacterial meningitis (defined as a glucose level of less than $34 \mathrm{mg} / \mathrm{dL}$ [1.9 mlol/ L], a ratio of CSF glucose to blood glucose of less than 0.23 , a protein level of more than $220 \mathrm{mg} / \mathrm{dL}$, or a leukocyte count of more than $2000 / \mu \mathrm{L}$ ) [19] and the clinical presentation was compatible with bacterial meningitis. In this study, the definitions of bacteremia, pneumonia and septic shock were also based on the Center of Disease Control [20]. We also evaluated the presences of neurological complications and long-term neurological sequelae of these patients, based on the definition of previous studies [12, 21].

\section{Capsular serotyping}

The capsule genotypes of all isolates were analyzed using the multiplex PCR approach, and this assay, as well as the DNA isolation method, was described in our previous publication [22].

\section{MLST assay and assignment to clonal clusters}

MLST was carried out as described in our previous study [23]. Briefly, PCR was used to amplify fragments from seven housekeeping genes (adhP, atr, glcK, $g \ln A$, pheS, $s d h A$, and $t k t$ ), and then seven PCR products were purified and sequenced. The sequence type (ST) was assigned based on the allelic profile of each fragment and determined via the Streptococcus agalactiae MLST database (http://pubmist.org/sagalactiae).

\section{Antimicrobial susceptibility testing}

Antimicrobial susceptibility testing was performed with the disc diffusion method as described in previous studies [24]. The double-disk diffusion test was applied to identify inducible clindamycin resistance. All GBS isolates were rated for susceptibility to seven antibiotics, 
including erythromycin, penicillin, clindamycin, vancomycin, ampicillin, cefotaxime and teicoplanin according to the guidelines of the Clinical and Laboratory Standards Institute for the microdilution minimum inhibitory concentration (MIC) method [25].

\section{Statistical analysis}

CPS distribution with respect to EOD or LOD, gender, and clinical characteristics including outcomes were compared using the $x^{2}$ test. To assess the differences between nominal variables, the Student's $t$ test or the Mann-Whitney $U$ test was used. The trend of proportions of the categorical variables among the three groups was analyzed by the Cochran-Armitage trend test. $P$ values of $<0.05$ were considered statistically significant. Data were analyzed using SPSS version 23 (IBM SPSS Statistics).

\section{Results}

\section{CPS distribution and clinical characteristics}

From January 2003 to December 2017, 182 nonduplicate GBS isolates from neonates with invasive GBS (iGBS) disease in CGMH were collected, and clinical characteristics were recorded. There were a total of 41 EOD (22.5\%), 121 LOD (66.5\%), and 20 LLOD (11.0\%) (Table 1). Serotypes III predominated in these invasive GBS strains $(n=118,64.8 \%)$, followed by serotype Ia $(n=33,18.1 \%)$ and Ib $(n=15,8.2 \%)$. Only 6 serotypes were detected during the 15-year study period surveyed. The majority of iGBS isolates (85.7\%) were submitted from blood specimens, and only a few isolates were collected from CSF (24 cases [13.2\%]). Both pleural fluid and intra-abdominal source each accounted for one case $(0.55 \%)$, respectively.

When comparing the distribution of serotypes in LOD and EOD, type III was significantly predominant in LOD (96 cases, $79.3 \%, p<0.001$ by $\chi^{2}$ test) and type Ib was significantly common in EOD (10 cases, 66.7\%). Other serotypes were similarly distributed. In this 15-year study period, we found a significant increase in serotype III in young infants with invasive GBS diseases (Fig. 1). Serotype III accounted for 37.5\% of all invasive GBS strains during 2003-2005, and increased to 78.2 and $74.5 \%$ of all invasive GBS isolates during 2010-2013 and 2014-2017, respectively (both $p<0.001$ ).

The clinical presentations of these invasive GBS diseases are summarized in Table 2. The majority of these neonates were term-born (gestational age [GA] more than 37 weeks, $79.1 \%$ [144/182]), and only 8 (4.4\%) were $\mathrm{GA}<30$ weeks. The median birth weight was $2900 \mathrm{~g}$ (interquartile range: $2557-3300 \mathrm{~g}$ ), and $57.7 \%$ were male. Of the total 182 cases, 12 (3 EOD, 7 LOD, and 2 LLOD) died before discharge, with a mortality rate of $6.6 \%$, and 29 (15.9\%) had neurological complications. Among those who survived and with neurological complications, 14 (45.4\%) had long-term neurological sequelae at discharge. Although serotype III GBS strains accounted for more than half of neonatal meningitis (31/51, 60.8\%), infants with type III GBS invasive disease were significantly less severe and had relatively better prognosis, including lower risk of neurological complications, less sepsis-attributable mortality and a higher rate of complete cure without any sequelae at discharge. On the contrary, infants with type Ib and type V GBS diseases had a significantly higher rates of respiratory failure, disseminated intravascular coagulopathy (DIC), and multiorgan failure (all $P$ values were $<0.05$ by $x^{2}$ test with Bonferroni correction) when compared with other serotypes. Furthermore, infants with type Ib and V GBS invasive disease had the highest risk of mortality (both $33.3 \%, P<0.001$ ).

All these isolates underwent MLST analyses. The MLST analyses revealed 11 different STs among the 182 GBS isolates. ST17 was the predominant ST and accounted for $56.6 \%$ of all isolates. All STs have been identified in the GBS MLST database (http://pubmist. org/sagalactiae). Relationships between STs and GBS serotypes of the 182 isolates characterized in this study are presented in Table 3. The correlation between serotype

Table 1 Numbers of invasive group B Streptococcal (iGBS) infections cases by capsular polysaccharide serotypes (CPS) and the clinical characteristics

\begin{tabular}{|c|c|c|c|c|c|c|c|}
\hline \multirow[t]{2}{*}{ CPS } & \multicolumn{3}{|c|}{ Age of iGBS onset } & \multicolumn{4}{|c|}{ Source of iGBS isolates } \\
\hline & EOD & LOD & LLOD & Blood & CSF & Ascites & $\overline{\text { Pleural fluid }}$ \\
\hline la & $10(24.4)$ & $19(15.7)$ & $4(20.0)$ & $27(17.3)$ & $6(25.0)$ & $0(0)$ & $0(0)$ \\
\hline $\mathrm{lb}$ & $10(24.4)$ & $2(1.7)$ & $3(15.0)$ & $13(8.3)$ & $2(8.3)$ & $0(0)$ & $0(0)$ \\
\hline$\|$ & $2(4.9)$ & $1(0.8)$ & $0(0)$ & $3(1.9)$ & $0(0)$ & $0(0)$ & $0(0)$ \\
\hline III & $14(34.1)$ & $96(79.3)$ & $8(40.0)$ & $102(65.4)$ & $16(66.7)$ & $0(0)$ & $0(0)$ \\
\hline V & $2(4.9)$ & $2(1.7)$ & $2(10.0)$ & $6(3.8)$ & $0(0)$ & $0(0)$ & $0(0)$ \\
\hline VI & $3(7.3)$ & $1(0.8)$ & $3(15.0)$ & $5(3.2)$ & $0(0)$ & $1(100)$ & $1(100)$ \\
\hline Total & $41(22.5)$ & $121(66.5)$ & $20(11.0)$ & $156(85.7)$ & $24(13.2)$ & $1(0.55)$ & $1(0.55)$ \\
\hline
\end{tabular}

EOD: early onset disease ( $\leq 7$ days); LOD: late onset disease (8-90 days); LLOD: late late-onset disease (> 90 days); CSF: cerebrospinal fluid 


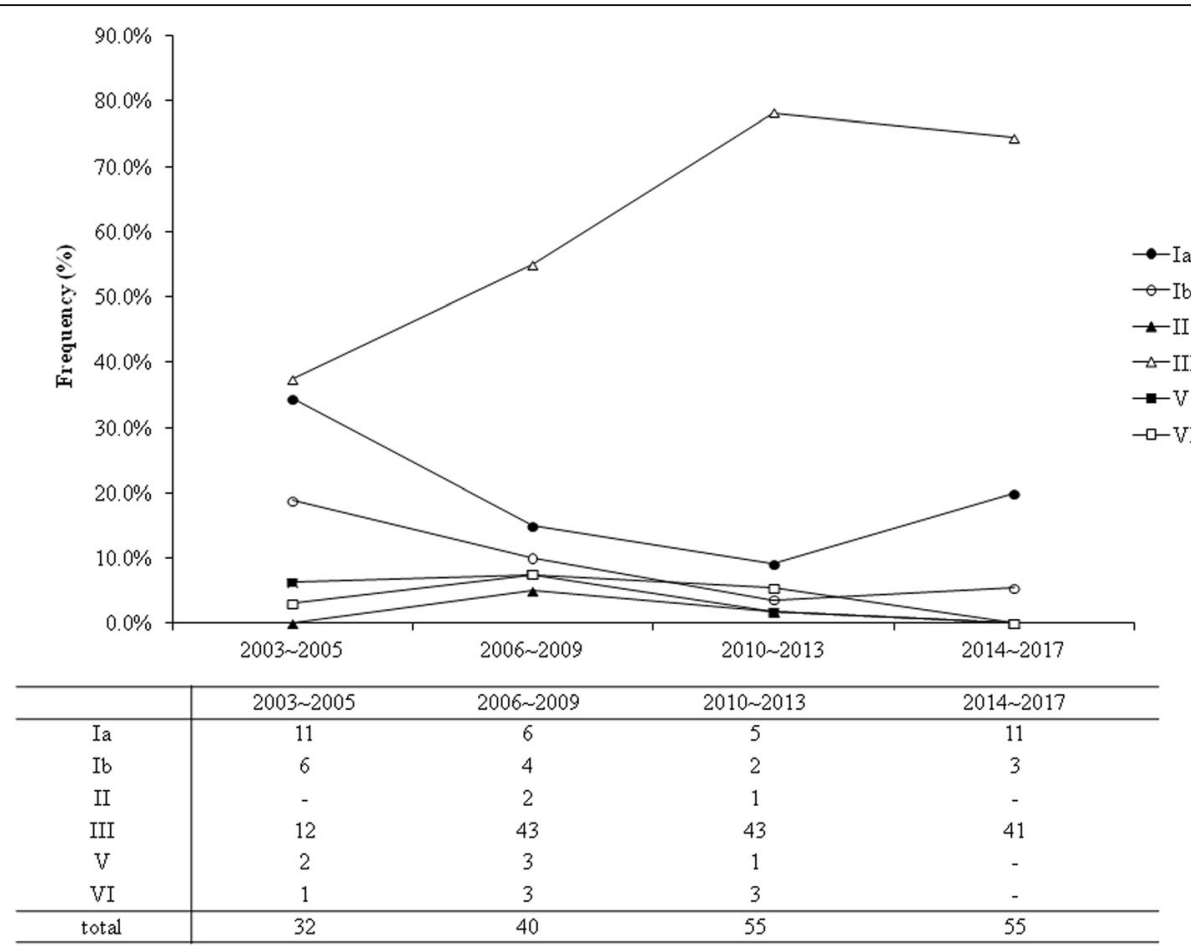

Fig. 1 The percentage of six serotypes invasive group B streptococcal disease in infants younger than one year old in Taiwan during a 15-year study period

III and ST17 was evident, as ST17 accounted for $87.3 \%$ $(n=103,95 \%$ confidence interval [CI] 86.6-88.0\%) of all serotype III isolates. The other 15 serotype III isolates belonged to ST19 $(11.0 \%, n=13)$, ST335 $(0.8 \%, \mathrm{n}=1)$, and ST438 $(0.8 \%, \mathrm{n}=1)$. Furthermore, the increasing trend of ST17 was also obvious, as we found that ST17 accounted for only $18.8 \%$ of all invasive GBS diseases during 2003-2005, and this strain increased to 69.1 and 74.5\% of all GBS isolates during 2010-2013 and 20142017, respectively (both $p<0.001$ ) (Fig. 2).

All isolates were susceptible to penicillin, ampicillin, and vancomycin. None of these strains displayed cefotaxime resistance. The overall rates of erythromycin and clindamycin resistance were 68.1 and $65.9 \%$, respectively. Most of the GBS isolates that were erythromycin resistant $(n=124,68.1 \%)$ were also clindamycin resistant (94.4\%, 117 isolates, $p<0.001$ by Pearson $\chi^{2}$ test). As we observed antimicrobial resistance profiles by serotype and ST, significantly higher rates of erythromycin and clindamycin resistances were noted in serotypes Ib (100\%), III (77.1-82.2\%) and V (66.7\%). There was no significant difference in the numbers of erythromycinnonsusceptible or clindamycin-nonsusceptible GBS isolates obtained from groups of EOD, LOD, or LLOD. Because the high correlation between ST17 and type III, and ST 12 and type Ib GBS strains, erythromycin resistance was found to be especially high among ST12 (100\%) and ST17 (89.3\%).

\section{Discussion}

Current studies of GBS have focused on the source, the bacterial factors influencing the transmission of GBS from mother to infants, and the predisposing factors for invasive GBS late-onset sepsis (LOS) in colonized infants [26-28]. Other studies have emphasized the need to develop GBS vaccines $[29,30]$. Therefore, all these studies highlighted the importance of preventive strategies and were based on epidemiological and molecular characteristics of GBS [24-31]. In this study, we also characterized the clinical manifestations of various invasive GBS strains in infants. We found a predominant and increasing trend of type III/ST-17 GBS strains in the past 15 years. In our cohort, although type III GBS strains accounted for more than half of neonatal meningitis, it appeared to be unrelated to the worst prognosis. On the contrary, type Ib and type V GBS diseases were associated with the highest illness severity and had the worst treatment outcomes. Type Ib and type III GBS strains had significantly higher resistance to erythromycin and clindamycin.

Serotype III GBS has become the most prevalent strain in Asia, accounting for $32.2-77.9 \%$ of all invasive GBS isolates in various countries and populations of specific age [32-36]. Although the distribution and predominance of certain serotypes are susceptible to variations and may change over time, there is an increasing trend of serotype III in infants [37, 38], which is also observed 
Table 2 Demographics and clinical features of neonates with serotype III GBS invasive infections compared with those with other serotype GBS invasive infections

\begin{tabular}{|c|c|c|c|c|c|}
\hline Clinical characteristics & $\begin{array}{l}\text { Type III iGBS } \\
(n=118)\end{array}$ & $\begin{array}{l}\text { Type la iGBS } \\
(n=33)\end{array}$ & $\begin{array}{l}\text { Type Ib iGBS } \\
(n=15)\end{array}$ & $\begin{array}{l}\text { Type V iGBS } \\
(n=6)\end{array}$ & $\begin{array}{l}\text { Other iGBS } \\
(n=10)\end{array}$ \\
\hline Gestational age (weeks), median (IQR) & $39.0(38.0-40.0)$ & $38.0(37.0-39.0)$ & $37.0(30.0-39.0)^{*}$ & $35.5(29.8-39.3)^{*}$ & $37.0(34.8-40.0)$ \\
\hline Birth body weight (g), median (IQR) & $\begin{array}{l}3040(2700- \\
3350.0)\end{array}$ & $\begin{array}{l}2760(2490- \\
3085)\end{array}$ & $\begin{array}{l}3015(1350- \\
3165)^{*}\end{array}$ & $\begin{array}{l}2248(1586- \\
2912)^{*}\end{array}$ & $\begin{array}{l}2560(2016- \\
3138)^{*}\end{array}$ \\
\hline Gender, male/female n (\%) & $77(65.3) / 41(34.8)$ & $\begin{array}{l}13(39.4) / 20 \\
(60.6)\end{array}$ & $8(53.3) / 7(46.7)$ & $4(66.7) / 2(33.3)$ & $5(50.0) / 5(50.0)$ \\
\hline Onset of diseases (day), median (IQR) & $27.5(14.8-53.3)$ & $30.0(3.5-56.5)$ & $2.0(1.0-30.0)^{*}$ & $14.0(1.0-21.3)^{*}$ & $2.5(1.0-11.8)^{*}$ \\
\hline Early-onset disease ( $\leq 7$ days) & $14(11.9)$ & $10(30.3)$ & $10(66.7)^{*}$ & $2(33.3)$ & $5(50.0)$ \\
\hline Late-onset disease (8-90 days) & $96(81.4)^{*}$ & $19(57.6)$ & $2(13.3)$ & $2(33.3)$ & $2(20.0)$ \\
\hline Late late-onset disease (> 90 days) & $8(6.8)$ & $4(12.1)$ & $3(20.0)$ & $2(33.3)$ & $3(30.0)$ \\
\hline \multicolumn{6}{|l|}{ Clinical manifestations } \\
\hline Bacteremia & $115(97.5)$ & $32(97.0)$ & $15(100)$ & $6(100)$ & $10(100)$ \\
\hline Meningitis & $31(26.3)$ & $13(39.4)$ & $6(40.0)$ & $0(0)$ & $1(10.0)$ \\
\hline Pneumonia & $12(10.2)$ & $2(6.1)$ & $5(33.3)$ & $0(0)$ & $2(20.0)$ \\
\hline Septic shock & $8(6.8)$ & $9(27.3)$ & $7(46.7)^{* *}$ & $3(50.0)^{* *}$ & $4(40.0)^{*}$ \\
\hline Respiratory failure & $8(6.8)$ & $8(24.2)$ & $9(60.0)^{* *}$ & $4(67.7)^{* *}$ & $4(40.0)^{*}$ \\
\hline Multi-organ failure & $2(1.7)$ & $3(9.1)$ & $7(46.7)^{* *}$ & $3(50.0)^{* *}$ & $4(40.0)^{*}$ \\
\hline Disseminated intravascular coagulopathy & $5(4.2)$ & $4(12.1)$ & $7(46.7)^{* * *}$ & $3(50.0)^{* *}$ & $4(40.0)^{*}$ \\
\hline \multicolumn{6}{|l|}{ Treatment outcomes } \\
\hline $\begin{array}{l}\text { Complete cure without any sequelae at } \\
\text { discharge }\end{array}$ & $104(88.1)$ & $26(78.8)$ & $8(53.3)^{* *}$ & $3(50.0)^{*}$ & $7(70.0)$ \\
\hline Neurological complications & $15(12.7)$ & $6(18.2)$ & $2(13.3)$ & $1(16.7)$ & $5(50.0)^{* *}$ \\
\hline Mortality & $1(0.8)$ & $3(9.1)$ & $5(33.3)^{* *}$ & $2(33.3)^{* *}$ & $1(10.0)$ \\
\hline
\end{tabular}

All data were expressed as number (percentage \%), unless indicated otherwise IQR: interquartile range

${ }^{*} P<0.05$ by $x^{2}$ test with Bonferroni correction

${ }^{*} P<0.001$ by $x^{2}$ test with Bonferroni correction

Table 3 Relationships between sequence type and serotype in 182 invasive GBS isolates in CGMH, 2003-2017

\begin{tabular}{|c|c|c|c|c|c|c|c|}
\hline \multirow{2}{*}{$\begin{array}{l}\text { Sequence } \\
\text { type }\end{array}$} & \multicolumn{7}{|c|}{ Serotype } \\
\hline & la (\%) & lb (\%) & || (\%) & III (\%) & V (\%) & VI (\%) & Total (\%) \\
\hline ST1 & 1 & - & 3 & - & 3 & 7 & $14(7.7)$ \\
\hline ST12 & - & 15 & - & - & 1 & - & $16(8.8)$ \\
\hline ST17 & - & - & - & 103 & - & - & 103 (56.6) \\
\hline ST19 & - & - & - & 13 & - & - & $13(7.1)$ \\
\hline ST23 & 16 & - & - & - & 2 & - & $18(9.9)$ \\
\hline ST24 & 10 & - & - & - & - & - & $10(5.5)$ \\
\hline ST144 & 1 & - & - & - & - & - & $1(0.5)$ \\
\hline ST268 & 2 & - & - & - & - & - & $2(1.1)$ \\
\hline ST335 & - & - & - & 1 & - & - & $1(0.5)$ \\
\hline ST438 & - & - & - & 1 & - & - & $1(0.5)$ \\
\hline ST890 & 3 & - & - & - & - & - & $3(1.6)$ \\
\hline Total (\%) & 33 & 15 & 3 & 118 & 6 & 7 & $182(100)$ \\
\hline
\end{tabular}

ST sequence type in our cohort. Furthermore, serotype III GBS is the most dominant invasive clone accounting for the majority of LOD cases with meningitis [35, 38, 39]. The most prevalent ST found in our cohort was ST17, followed by ST23 and ST12. The common ST19 strain found in other countries only accounted for $7.1 \%$ of our isolates. In colonized pregnant women and adults with invasive disease, most type III GBS isolates belong to ST-17 and ST-19 $[35,39,40]$; however, serotype III GBS strains that cause invasive disease in infants are almost exclusively ST-17 lineage. These results indicate that neonatal invasive diseases may not be directly derived from maternal colonization, and the transmission routines of GBS LOD, especially those occur later than 90 days of age (late LOD), remain unknown.

In this present study, it is noteworthy that type Ib, type V and type VI GBS strains were associated with more severe clinical presentations, including higher rates of septic shock, disseminated intravascular coagulopathy, and multi-organ failure, leading to a higher mortality. Besides, these strains were associated with a higher rate of neurological complications, although only type Ib GBS 


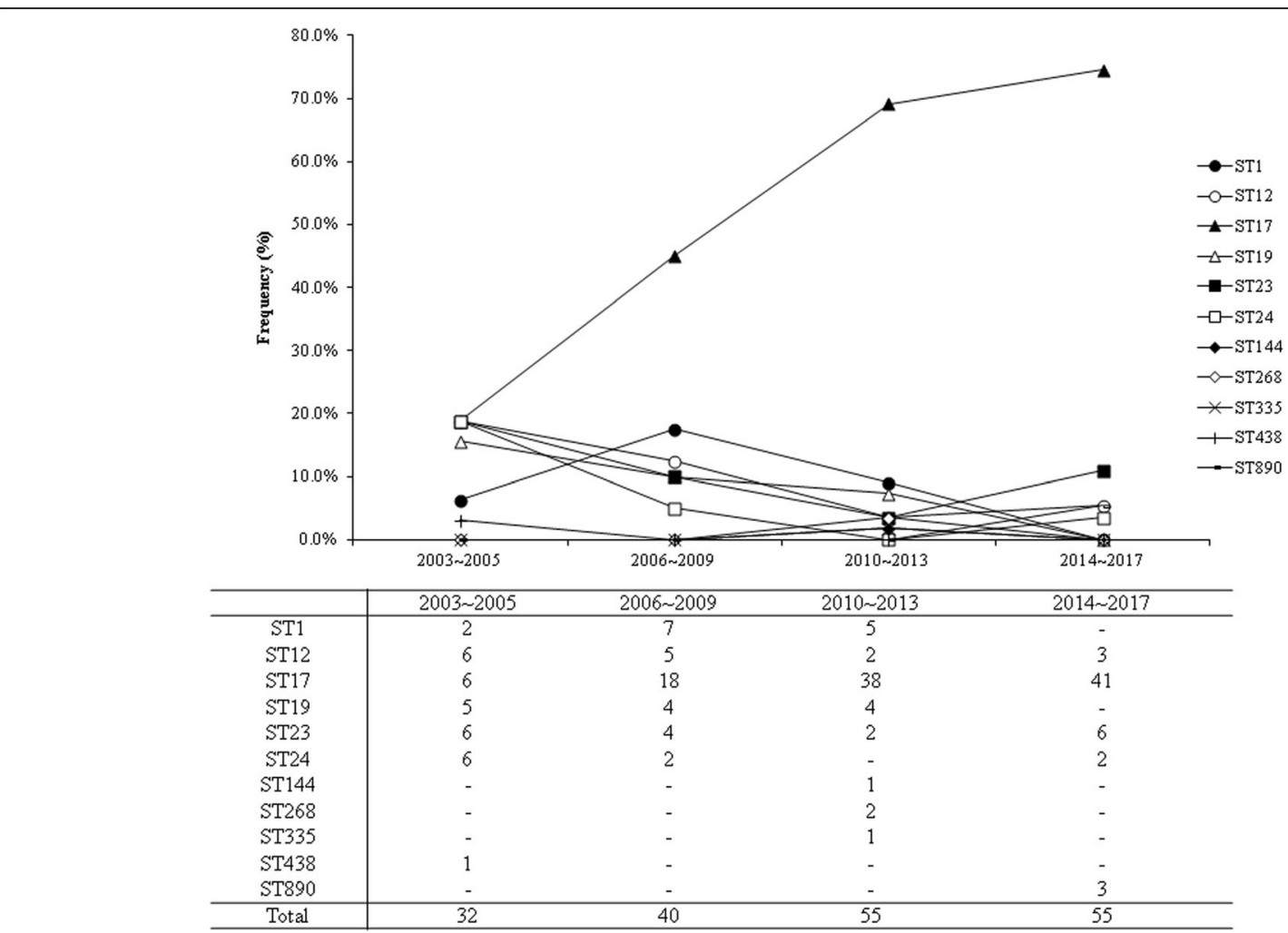

Fig. 2 Invasive group B streptococcal disease in young infants younger than one year old in Taiwan, 2003-2017, stratified based on different sequence types and time period

strain often caused meningitis. These complications resulted from poor cerebral perfusion during septic shock rather than sequelae related to meningitis. The higher mortality rate of type V GBS resulted from the lower gestational age ( 3 out of 6 were preterm infants, including 2 less than 28 weeks). On the contrary, although type III GBS strains were associated with a higher rate of meningitis, most were completely cured at discharge. Few studies have characterized the clinical manifestations of invasive GBS diseases in a specific serotype or sequence type, but our findings were limited by small sample sizes. Therefore, further systemic reviews or multi-center studies are required to conclude these clinical features.

The overall mortality in our cohort was $6.6 \%$, which was lower than the average mortality of $8.4 \%$ (95\% confidence interval 6.6-10.2) reported by other countries [11], including Japan, Korea, India, and a recent study in South China [30, 36, 41-43]. In our cohort, 28\% (total $n=51$ ) had CSF culture proven or clinical meningitis, which was significantly higher than previous reports [11, 36, 41-43]. Furthermore, the presence of neurological sequelae after treatment in our cohort (16\%) was significantly lower than the reported $25-35 \%$ in previous studies $[43,44]$. These may result from different definitions of meningitis and neurological sequelae. Because none of these isolates were resistant to penicillin and none had delayed administration of effective antibiotic when disease onset, further study to explore optimized therapeutic strategies will be necessary given the remarkable adverse outcomes in these patients.

The rates of GBS resistance to macrolide and clindamycin have been increasing in a global trend since the 2000s $[45,46]$. It is noteworthy that GBS isolates resistant to vancomycin and gentamicin have recently been reported in some geographies [47, 48]. In this cohort, certain GBS serotypes and STs were significantly associated with erythromycin and clindamycin resistance. The increasing trend of GBS resistance to macrolide and clindamycin in our cohort is associated with the trend of type III/ST-17 GBS isolates. Furthermore, type Ib GBS has the highest rate of antimicrobial resistance, which is in consistent with that reported in Southeast Asia [47-49]. GBS isolates in Taiwan and China have been found to have a higher rate of macrolide resistance (58$70.8 \%$ ), when compared with the resistant rate of $11.5-36 \%$ reported in Europe and North America [11, 37, 40, 41, 49-52]. These findings add concerns regarding the prophylactic use of ampicillin in pregnant women, as antibiotic selection may account for the increased antibiotic resistance, which finally lead 
to limited therapeutic choices for penicillinintolerant patients.

This study has some limitations. All GBS isolates were from a single center in Taiwan, and may not represent the whole epidemiological features of this geographic area. Most of our iGBS strains were type III/ST-17, and other serotypes, especially type II, V and VI were small case numbers. Therefore, we were unable to characterize the clinical manifestations of specific serotype GBS invasive diseases. The slide agglutination was not performed in this study, so the expression of genes detected was unknown. Furthermore, although we found that the increasing trend of type III/ST-17 GBS strains is causing invasive diseases in infants, we did not have data of population-based incidence rate. Finally, some false-negative cases or early mortality caused by GBS infection might have been missed and were not included in this study.

\section{Conclusions}

In conclusion, this study demonstrates that GBS remains one of the most important pathogens that cause neonatal mortality and morbidity, especially those with meningitis and neurological sequelae. There is an increasing trend of type III/ST-17 GBS in Taiwan, and the increasing trends of antibiotic resistance to erythromycin and clindamycin were also noted in Taiwan, and in Asia. This is of great concern, because the antibiotic options for penicillin-allergic women will be limited. Furthermore, we used to have initial appropriate antibiotic treatment in time, but the adverse outcomes of invasive GBS diseases are sometimes inevitable. Therefore, further study on more aggressive treatments and monitoring will be necessary in the future.

\section{Abbreviations}

CGMH: Chang Gung Memorial Hospital; CPS: Capsular polysaccharides serotypes; CSF: Cerebrospinal fluid; EOD: Early-onset disease; GBS: Group B streptococcus; LLOD: Late late-onset disease; LOD: Late-onset disease: MLST: Multilocus sequence typing; ST: Sequence type

\section{Acknowledgements}

All authors thank Mrs. Chiao-Ching Chiang for keeping the database of our $\mathrm{NICU}$, and all nursing staff working in our NICUs for keeping extremely detailed patient records, which contributed greatly to the completion of this research.

\section{Authors' contributions}

Conceptualization: YK, MHT, MYL. Data collection and verification: YK, MHT, SMC, HRH, MCC, RHF. Formal analysis: YK, MHT, SMC. Funding acquisition: JFH, JJL. Investigation: YK, MHT, MCC. Methodology: JFH, MYL, SMC, HRH. Supervision: JJL and JFH. Writing-original drift: YK and MHT. Writing-review \& editing: JJL and JFH. All authors have read and approved the final version of this manuscript.

\section{Funding}

This work was supported by grants from Chang Gung Memorial Hospital (CMRPG3H0191 and CMRPG3E1491) and Ministry of Science and Technology Taiwan (MOST-105-2314-B-182-065-MY2).

Role of funder: Both the Chang Gung Memorial Hospital and Ministry of Science and Technology provided the budget to complete the molecular studies in this manuscript.

\section{Availability of data and materials}

The datasets used/or analyzed during the current study available from the corresponding author on reasonable request.

Ethics approval and consent to participate

This study was approved by the institutional review board of Chang Gung Memorial Hospital, with a waiver of informed consent because all patients records and information were anonymized and de-identified prior to analysis.

Consent for publication

Not applicable.

\section{Competing interests}

The authors declare that they have no competing interests.

\section{Author details}

'Division of Pediatric Neonatology, Department of Pediatrics, Chang Gung Memorial Hospital, Taoyuan, Taiwan. ${ }^{2}$ Division of Neonatology and Pediatric Hematology/Oncology, Department of Pediatrics, Chang Gung Memorial Hospital, Yunlin, Taiwan. ${ }^{3}$ Department of Laboratory Medicine, Chang Gung Memorial Hospital at Linkou, Taoyuan, Taiwan. ${ }^{4}$ College of Medicine, Chang Gung University, Taoyuan, Taiwan. ${ }^{5}$ Department of Medical Biotechnology and Laboratory Science, Chang Gung University, Taoyuan, Taiwan. ${ }^{6}$ Division of Neonatology, Department of Pediatrics, Chang Gung Memorial Hospital, 5, Fu-Shing St., Kwei-Shan, Taoyuan 333, Taiwan.

Received: 30 January 2019 Accepted: 10 June 2019

Published online: 19 June 2019

\section{References}

1. Lu B, Li D, Cui Y, Sui W, Huang L, Lu X. Epidemiology of group B streptococcus isolated from pregnant women in Beijing, China. Clin Microbiol Infect. 2014;20:0370-3.

2. Stokholm J, Schiørring S, Eskildsen CE, Pedersen L, Bischoff AL, Følsgaard N, et al. Antibiotic use during pregnancy alters the commensal vaginal microbiota. Clin Microbiol Infect. 2014:20:629-35.

3. Madrid L, Maculuve SA, Vilajeliu A, Sáez E, Massora S, Cossa A, et al. Maternal carriage of group B Streptococcus and Escherichia coli in a Distric Hospital in Mozambique. Pediatr Infect Dis J. 2018;37:1145-53.

4. Russell NJ, Seale AC, O'Driscoll M, O'Sullivan C, Bianchi-Jassir F, GonzalezGuarin J, et al. Maternal colonization with group B Streptococcus and serotype distribution worldwide: systemic review and meta-analyses. Clin Infect Dis. 2017:65:S100-11.

5. van Kassel MN, Bijisma MW, van der Ende A, Brouwer MC, van de Beek D. Community acquired group B streptococcal meningitis in adults: 33 cases from prospective cohort studies. J Inf Secur 2018; Jul 28 [Epub ahead of print].

6. Joubrel C, Tazi A, Six A, Dmytruk N, Touak G, Bidet P, et al. Group B streptococcus neonatal invasive infections, France 2007-2012. Clin Microbiol Infect. 2015;21:910-6.

7. Ouchenir L, Renaud C, Khan S, Bitnun A, Boisvert AA, McDonald J, et al. The epidemiology, management, and outcomes of bacterial meningitis in infants. Pediatrics. 2017;140(1):e20170476.

8. Metcalf BJ, Chochua S, Gertz RE Jr, Hawkins PA, Ricaldi J, Li Z, et al. Shortread whole genome sequencing for determination of antimicrobial resistance mechanisms and capsular serotypes of current invasive Streptococcus agalactiae recoved in the USA. Clin Microbiol Infect. 2017; 23(8):574.e7-574.e14

9. Melin P. Neonatal group B streptococcal disease: from pathogenesis to preventive strategies. Clin Microbiol Infect. 2011;17:1294-303.

10. Serenius F, Ewald U, Faroogi A, Fellman V, Hafstrom M, Hellgren $\mathrm{K}$, et al. Neurodevelopmental outcomes among extremely preterm infants 6.5 years after active perinatal care in Sweden. JAMA Pediatr. 2016;170(10):954-63.

11. Madrid L, Seale AC, Kohil-Lynch M, Edmond KM, Lawn JE, Heath PT, et al. Infant group B streptococcal disease incidence and serotypes worldwide: systemic review and meta-analyses. Clin Infect Dis. 2017:65(Suppl 2):S160-72.

12. Hsu MH, Hsu JF, Kuo HC, Lai MY, Chiang MC, Lin YJ, et al. Neurological complications in young infants with acute bacterial meningitis. Front Neurol. 2018;9:903.

13. Medugu N, Iregbu KC, Parker RE, Plemmons J, Singh P, Audu LI, et al. Group $B$ streptococcal colonization and transmission dynamics in pregnant 
women and their newborns in Nigeria: implications for prevention strategies. Clin Microbiol Infect. 2017;23(9):673.e9-e16.

14. Mukhopadhyay S, Dukhovny D, Mao W, Eichenwald EC, Puopolo KM. 2010 perinatal GBS prevention guideline and resource utilization. Pediatrics. 2014; 133:196-203.

15. Berardi A, Rossi C, Guidotti I, Vellani G, Lugli L, Bacchi Reggiani ML, et al. Factors associated with intrapartum transmission of group B Streptococcus. Pediatr Infect Dis J. 2014;33:1211-5.

16. Ellem JA, Kovacevic D, Olma T, Chen SC. Rapid detection of group B streptococcus directly from vaginal-rectal specimens using liquid swabs and the BD max GBS assay. Clin Microbiol Infect. 2017;23:948-51.

17. van der Mee-Marquet N, Diene SM, Barbera L, Courtier-Martinez L, Lafont $L$, Ouachee A, et al. Analysis of the prophages carried by human infecting isolates provides new insight into the evolution of group B Streptococcus species. Clin Microbiol Infect. 2018;24:514-21.

18. van de Beek D, Cabellos C, Dzupova O, Esposito S, Klein M, Kloek AT, et al. ESCMID guideline: diagnosis and treatment of acute bacterial meningitis. Clin Microbiol Infect. 2016;22:S37-62.

19. African Neonatal Sepsis Trial (AFRINEST) group, Tshefu A, Lokangaka A, Ngaima S, Engmann C, Esamai F, Gisore P, et al. Simplified antibiotic regimens compared with injectable procaine benzylpenicillin plus gentamicin for treatment of neonates and young infants with clinical signs of possible serious bacterial infection when referral is not possible: a randomized, open-label, equivalence trial. Lancet. 2015;385:1767-76.

20. Vergnano S, Buttery J, Cailes B, Chandrasekaran R, Chiappini E, Clark E, et al. Neonatal infections: case definition and guidelines for data collection, analysis, and presentation of immunization safety data. Vaccine. 2016:34:6038-46.

21. Jim KK, Brouwer MC, van der Ende A, van de Beek D. Subdural emphyme in bacterial meningitis. Neurology. 2012;79:2133-9.

22. Tien N, Ho CM, Lin HJ, Shih MC, Ho MW, Lin HC, et al. Multilocus sequencing typing of invasive group B streptococcus in central area of Taiwan. J Microbiol Immunol Infect. 2011:44(6):430-4.

23. Manning SD, Springman AC, Lehotzky E, Lewis MA, Whittam TS, Davies HD. Multilocus sequence types associated with neonatal group B streptococcal sepsis and meningitis in Canada. J Clin Microbiol. 2009;47(4):1143-8.

24. Matuschek E, Ahman J, Webster C, Kahlmeter G. Antimicrobial susceptibility testing of colistin-evolution of seven commercial MIC products against standard broth microdilution for Escherichia coli, Klebsiella pneumonia, Pseudomonas aeruginosa, and Acinetobacter spp. Clin Microbiol Infect. 2018:24(8):865-70.

25. Clinical and Laboratory Standards Institute. Performance standards for antimicrobial susceptibility testing, twenty-fourth informational supplement, M100-S24. SLCl, Wayne, Ml; 2014.

26. Seedat F, Brown CS, Stinton C, Patterson J, Geppert J, Freeman K, et al. Bacterial load and molecular markers associated with early-onset group B Streptococcus: a systemic review and meta-analysis. Pediatr Infect Dis J. 2018 Mar 27; Epub ahead of print.

27. Al Luhidan L, Madani A, Albanyan EA, Al Saif S, Nasef M, AlJohani S, et al. Neonatal Group B Streptococcal Infection in a Tertiary Care Hospital in Saudi Arabia: a 13-year Experience. Pediatr Infect Dis J. 2019;38:731-34.

28. Roca A, Bojang A, Camara B, Oluwalana C, Lette K, West P, et al. Maternal colonization with Staphylococcus aureus and group $B$ streptococcus is associated with colonization in newborns. Clin Microbiol Infect. 2017;23(12):974-9.

29. Tsai MH, Hsu JF, Lai MY, Lin LC, Chu SM, Huang HR, et al. Molecular Characteristics and Antimicrobial Resistance of Group B Streptococcus Strains Causing Invasive Disease in Neonates and Adults. Front Microbiol. 2019;10:264.

30. Li S, Wen G, Cao X, Guo D, Yao Z, Wu C, et al. Molecular characteristics of Streptococcus agalactiae in a mother-baby prospective cohort study: implication for vaccine development and insights into vertical transmission. Vaccine. 2018;36(15):1941-8.

31. Metcalf BJ, Chochua S, Gertz RE Jr, Hawkins PA, Ricaldi J, Li Z, et al. Shortread whole genome sequencing for determination of antimicrobial resistance mechanisms and capsular serotypes of current invasive Streptococcus agalactiae recovered in the USA. Clin Microbiol Infect. 2017; 23(8):574.e7-574 e14.

32. Jiang $H$, Chen M, Li T, Liu H, Gong Y, Li M. Molecular characterization of Streptococcus agalactiae causing community- and hospital- acquired infections in Shanghai, China. Front Microbiol. 2016;7:1308.

33. Creti R, Imperi M, Berardi A, Pataracchia M, Recchia S, Alfarone G, et al. Neonatal group B streptococcus infections: prevention strategies, clinical and microbiological characteristics in 7 years of surveillance. Pediatr Infect Dis J. 2017; 36(3):256-62.

34. Kang HM, Lee HJ, Lee H, Jo DS, Lee HS, Kim TS, et al. Genotype characterization of group B Streptococcus isolated from infants with invasive diseases in South Korea. Pediatr Infect Dis J. 2017;36(10):e242-7.

35. Shabayek S, Spellerberg B. Group B streptococcal colonization, molecular characteristics, and epidemiology. Front Microbiol. 2018;9:437.

36. Guan X, Mu X, Ji W, Yuan C, He P, Zhang L, et al. Epidemiology of invasive group $B$ streptococcal disease in infants from urban area of South China, 2011-2014. BMC Infect Dis. 2018;18(1):14.

37. Lamagni TL, Keshishian C, Efstratiou A, Guy R, Henderson KL, Broughton K, et al. Emerging trends in the epidemiology of invasive group $B$ streptococcal disease in England and Wales, 1991-2010. Clin Microbiol Infect. 2013;57(5):682-8.

38. Florindo C, Damiao V, Silvestre I, Farinha C, Rodrigues F, Nogueira F, et al. Epidemiological surveillance of colonising group B Streptococcus epidermiology in the Lisbon and Tagus Valley regions, Portugal (2005 to 2012): emergence of a new epidemic type IV/clonal complex 17 clone. Euro Surveill. 2014;19(23):20825.

39. Alhhazmi A, Hurteau D, Tyrrell GJ. Epidemiology of invasive group $B$ streptococcal disease in Alberta, Canada, from 2003 to 2013. J Clin Microbiol. 2016;54(7):1774-81.

40. Teatero S, Ferrieri P, Martin I, Demczuk W, McGeer A, Fittipaldi N. Serotype distribution, population structure, and antimicrobial resistance of group $B$ Streptococcus strains recovered from colonized pregnant women. J Clin Microbiol. 2017:55(2):412-22.

41. Park KH, Kim KH, Kang JH, Kim KN, Kim DS, Kim YK, et al. Current status and clinical presentations of invasive neonatal group B streptococcal infections in Korea. Pediatr Int. 2011:53:236-9.

42. Matsubara K, Hoshina K, Suzuki Y. Early-onset and late-onset group B streptococcal disease in Japan: a nationwide surveillance study, 2004-2010. Int J Infect Dis. 2013;17:e379-84.

43. Sridhar S, Grace R, Nithya PJ, Balaji V, Niranjan T, Manish K, et al. Group B streptococcal infection in a tertiary hospital in India - 1998-2010. Pediatr Infect Dis J. 2014;33:1091-2.

44. Tibussek D, Sinclair A, Yau I, Teatero S, Fittipaldi N, Richardson SE, et al. Lateonset group B streptococcal meningitis has cerebrovascular complications. J Pediatr. 2015;166:1187-92 e.1.

45. Da Cunha V, Davies MR, Douarre PE, Rosinski-Chupin I, Margarit I, Spinali S, et al. Streptococcus agalactiae clones infecting humans were selected and fixed through the extensive use of tetracycline. Nat Commun. 2014;5:4544.

46. Sendi P, Furitsch M, Mauerer S, Florindo C, Kahl BC, Shabayek S, et al. Chromosomally and extrachromosomally mediated high-level gentamicin resistance in Streptococcus agalactiae. Antimicrob Agents Chemother. 2016; 60(3):1702-7.

47. Park C, Nichols M, Schrag SJ. Two cases of invasive vancomycin-resistant group B streptococcus infection. N Engl J Med. 2014;370(9):885-6.

48. Guo D, Cao X, Li S, Ou Q, Lin D, Yao Z, et al. Neonatal colonization of group B Streptococcus in China: prevalence, antimicrobial resistance, serotypes, and molecular characterization. Am J Infect Control. 2018;46(3):e19-24.

49. Martins ER, Pedroso-Roussado C, Melo-Cristino J, Ramirez M. Portuguese group for the study of streptococcal infection. Streptococcus agalactiae causing neonatal infections in Portugal (2005-2015): diversification and emergence of a CC-17/PI-2b multidrug resistant sublineage. Front Microbiol. 2017;8:499.

50. Wang YH, Su LH, Hou JN, Yang TH, Lin TY, Chu C, et al. Group B streptococcal disease in nonpregnant patients: emergence of highly resistant strains of serotype lb in Taiwan in 2006 to 2008. J Clin Microbiol. 2010;48(7):2571-4.

51. Hawkins PA, Law CS, Metcalf BJ, Chochua S, Jackson DM, Westblade LF, et al. Cross-resistance to lincosamides, streptogramins a and pleuromutilins in Streptococcus agalactiae isolates from the USA.J Antimicrob Chemother. 2017;72(7):1886-92.

52. Campisi E, Rosini R, Ji W, Guidotti S, Rojas-Lopez M, Geng G, et al. Genomic analysis reveals multi-drug resistance clusters in group B Strepococccus CC17 hypervirulent isolates causing neonatal invaisve disease in southern mainland China. Front Microbiol. 2016;7:1265.

\section{Publisher's Note}

Springer Nature remains neutral with regard to jurisdictional claims in published maps and institutional affiliations. 